\title{
A Review of Silver Nanoparticles: Synthesis Methods, Properties and Applications
}

\author{
Jun Natsuki ${ }^{1}$, Toshiaki Natsuki ${ }^{1,2}$, Yoshio Hashimoto ${ }^{1,3}$ \\ ${ }^{1}$ Institute of Carbon Science and Technology, Shinshu University, Wakasato, Nagano-shi, Nagano, Japan \\ ${ }^{2}$ Faculty of Textile Science and Technology, Shinshu University, Tokida, Ueda-shi, Nagano, Japan \\ ${ }^{3}$ Faculty of Engineering, Shinshu University, Wakasato, Nagano, Japan \\ Email address: \\ jnatsu@shinshu-u.ac.jp (J. Natsuki),natsuki@shinshu-u.ac.jp (T. Natsuki), hashimt@shinshu-u.ac.jp (Y. Hashimoto)
}

\section{To cite this article:}

Jun Natsuki, Toshiaki Natsuki, Yoshio Hashimoto. A Review of Silver Nanoparticles: Synthesis Methods, Properties and Applications. International Journal of Materials Science and Applications. Vol. 4, No. 5, 2015, pp. 325-332. doi: 10.11648/j.ijmsa.20150405.17

\begin{abstract}
In recent years, nanoparticles of noble metals such as gold, silver and palladium have drawn immense attention due to the wide range of new applications in various fields of industry. Particularly, silver nanoparticles have significant interest in medical applications such as very effective antibacterial agents without the toxic effects, and industry application such as inkjet inks containing well uniform dispersions of nano-sized silver particles that are useful for producing electronic circuits. It is important that the silver nanoparticles require not only the particles to be of nano-size, but also synthesis of the nanoparticles to be produced easily and at low cost. Over the past few decades, many synthetic methods of silver nanoparticles have been studied. This paper aims to review different synthesis routes of silver nanoparticles and their applications. In particular, we mainly present several chemical approaches to preparing silver nanoparticles and their properties as well as applications based on our recent studies. The focus is on effective and efficient synthesis of pure colloidal silver nanoparticles with high electrical conductivity and their potential application.
\end{abstract}

Keywords: Sliver Nanoparticles, Synthesis, Electrical Conductivity, Electronic Circuits, Applications

\section{Introduction}

The application of nanoscale materials and structures is an emerging area of nanoscience and nanotechnology. Nano-sized metal particles have been used widely in various fields including catalysis and photonics. Among various metals, silver nanoparticles (AgNPs) are of particular interest due to their remarkable antimicrobial and localized surface plasmon resonance properties, which render them unique properties such as broad-spectrum antimicrobial [1-3], surface-enhanced Raman spectroscopy (SERS) [4,5], chemical /biological sensors and biomedicine materials [6-8], biomarker [9-11] and so on. Silver nanoparticles are usually ranging from 1 to 100 $\mathrm{nm}$ in size. They have unique optical, electrical, and thermal properties and are incorporated into industrial application of electronics, catalysis, and photonics. In recent years, with higher integrated density of electronic components, there are growing demands for the thickness or the width of printed electronic circuits due to considering the space between these circuits [12-14]. Therefore, the synthesis of AgNPs becomes an important issue in the electronic industry.
Currently, many methods and approaches have been reported for the synthesis of AgNPs by using chemical, physical, photochemical and biological routes. Each method has advantages and disadvantages with common problems being costs, scalability, particle sizes and size distribution and so on [15-19]. Physical and photochemical methods to prepare nanoparticles are usually need the very high temperature and vacuum conditions, and expensive equipment [20-22]. Among the existing methods, the chemical methods have been mostly used for production of AgNPs. It is well known that chemical method can successfully produce pure, well-defined nanoparticles and is also the most common method because of its convenience and simple equipment. Chemical methods provide an easy way to synthesize AgNPs in solution, because they can be implemented under simple and mild conditions. In chemical approach, the preparation of AgNPs with colloidal dispersions in water or organic solvents is performed by chemical reduction. The reduction of silver ions in solvents yields colloidal silver nanoparticles with different particle diameters. 
An increasingly common application of AgNPs can be found use in electronics industry. For example, inks, pastes and filler utilize AgNPs for their high electrical conductivity; molecular diagnostics and photonic devices take advantage of the novel optical properties of AgNPs [23-25]. In the manufacture of electronic circuits, sintering of nanoparticles is necessary to remove dispersing agents for obtaining high electrical conductivity and can usually be accelerated by heating. From an industrial point of view, it is necessary to develop simple and low-cost processes to produce large quantities of silver nanoparticles. It is preferable to perform sintering of nanopaticles at the lowest temperature possible [26-28].

In this review, we present different methods to prepare AgNPs and their properties as well as applications. In particular, we describe several novel methods based on our recent studies, which are successful in the synthesis of AgNPs with high conductive properties. We also describe the reaction mechanism of AgNPs and factors affecting particle size. The focus is on effective and efficient synthesis of pure colloidal silver nanoparticles with high electroconductivity, low cost and environment-friendly.

\section{Syntheses of Silver Nanoparticles and Their Property}

\subsection{Physical Approach}

In physical processes, metal nanoparticles are generally synthesized by evaporation condensation, which could be carried out using a tube furnace at atmospheric pressure. The source material within a boat centered at the furnace is vaporized into a carrier gas. Nanoparticles of various materials, such as $\mathrm{Ag}, \mathrm{Au}, \mathrm{PbS}$ and fullerene, have previously been produced using the evaporation/condensation technique [29-31]. However, the generation of AgNPs using a tube furnace has several drawbacks, because a tube furnace occupies a large space, consumes a great deal of energy while raising the environmental temperature around the source material, and requires a lot of time to achieve thermal stability. A typical tube furnace requires power consumption of more than several kilowatts and a preheating time of several tens of minutes to attain a stable operating temperature. Furthermore, silver nanoparticles have been synthesized with laser ablation of metallic bulk materials in solution [32-34]. One advantage of laser ablation compared to other conventional method for preparing metal colloids is the absence of chemical reagents in solutions. Therefore, pure colloids, which will be useful for further applications, can be produced by this method [35].

In summary, the physical synthesis of AgNPs usually utilizes the physical energies to produce AgNPs with nearly narrow size distribution. The physical approach can permit producing large quantities of AgNPs samples in a single process. This is also the most useful method to produce AgNPs powder. However, primary costs for investment of equipment should be considered.

\subsection{Photochemical Approach}

The photo-induced synthetic strategies have also been developed. For example, Huang and Yang synthesized AgNPs via photoreduction of $\mathrm{AgNO}_{3}$ in layered inorganic clay suspensions, which serves as stabilizing agent that prevent nanoparticles from aggregation. Irradiation disintegrated the AgNPs into smaller size with a single mode distribution until a relatively stable size and diameter distribution were achieved [36]. However, in this method, the equipments with high cost and experimental environment are required.

\subsection{Biological Approach}

Recently, biosynthetic methods using naturally reducing agents such as polysaccharides, biological microorganism such as bacteria and fungus or plants extract, i.e. green chemistry, have emerged as a simple and viable alternative to more complex chemical synthetic procedures to obtain AgNPs. Bacteria are known to produce inorganic materials either intra- or extracellularly. This makes them potential biofactories for the synthesis of nanoparticles like gold and silver. Particularly, silver is well known for its biotical properties.

A. R. Vilchis-Nestor et al. used green tea (Camellia sinensis) extract as reducing and stabilizing agent to produce gold silver nanoparticles in aqueous solution at ambient conditions [37]. Moreover, K. Kalishwaralal et al. reported the synthesis of AgNPs by reduction of aqueous $\mathrm{Ag}+$ ions with the culture supernatant of Bacillus licheniformis [38]. The synthesized AgNPs are highly stable and this method has advantages over other methods as the organism used here is a nonpathogenic bacterium. The biological method provides a wide range of resources for the synthesis of AgNPs, and this method can be considered as a method of nanoparticles synthesis with advantages over conventional chemical routes of synthesis and as an environmentally friendly approach as well as a low cost technique. However, it is not easy to obtain a large quantity of AgNPs by using biological synthesis.

\subsection{Chemical Approach}

Besides those approaches described above, chemical reduction is the most common method because of its convenience and simple equipment. Control over the growth of metal nanoparticles is required to obtain nanoparticles of small size with a spherical shape and narrow distribution in diameter. It is well known that silver nanoparticles can be produced by chemical reaction at low cost and in high yield. In this review we describe sevorious chemical synthesis methods to prepare the silver nanoparticles mainly.

Generally, the chemical synthesis process of AgNPs in solution usually employs the following three main components: (1) metal precursors, (2) reducing agents and (3) stabilizing/capping agents. The formation of colloidal solutions from the reduction of silver salts involves two stages of nucleation and subsequent growth. It is also revealed that the size and the shape of synthesized AgNPs are 
strongly dependent on these stages. Furthermore, for the synthesis of monodispered AgNPs with uniform size distribution, all nuclei are required to form at the same time. In this case, all the nuclei are likely to have the same or similar size, and then they will have the same subsequent growth. The initial nucleation and the subsequent growth of initial nuclei can be controlled by adjusting the reaction parameters such as reaction temperature, $\mathrm{pH}$, precursors, reduction agents (i.e. $\mathrm{NaBH}_{4}$, ethylene glycol, glucose) and stabilizing agents (i.e. PVA, PVP, sodium oleate) [39-41].

\subsubsection{Synthesis of AgNPs by Previous Research}

Many approaches to preparing AgNPs including chemical, physical and biological methods have been reported. Chemical reduction is the most common method because of its convenience and simple equipment. The intrinsic properties of the synthesized AgNPs are mainly determined by their size, shape and crystal structure, which can be controlled during chemical synthesis. In general, the chemical synthesis process of AgNPs uses reducing agents and stabilizing or capping agents and the size and distribution of AgNPs were influenced by reducing agents and stabilizers. The formation of colloidal solutions from the reduction of silver salts involves two stages of nucleation and subsequent growth [42].

D. Radziuk et al. prepared AgNPs by reduction of silver nitrate in an excess of aqueous sodium borohydride which be used as a reducing agent. The nanoparticles with a diameter of $20 \mathrm{~nm}$ are obtained [43]. C. Luo et al. reported AgNPs prepared under mild conditions by exploiting polyethylene glycol (PEG), and the dramatic effect of PEG to the formation of AgNPs [44]. The PEG is able to act as reducing agent and very sensitive to its molecular weight. The reducing rate of $\mathrm{Ag}+$ to nano- $\mathrm{Ag}$ was remarkably enhanced with the increase of polymer chain length of PEG. S. L. C. Hsu et al. synthesized AgNPs suspensions by chemical reduction from silver nitrate in a formaldehyde reductant and polyvinylpyrrolidone (PVP) stabilizer using organic bases as the reaction promoter [45]. The reduction process proceeded at relatively high temperature, and the complete separation of AgNPs was not easy because of the presence of other compounds in the reaction mixture. Nearly monodisperse AgNPs were prepared using a simple oleylamine-liquid paraffin system [46]. They found that the formation process of AgNPs could be divided into three stages: growth, incubation, and Ostwald ripening stages. The synthetic approach was developed to use liquid paraffin as the solvent instead of toluene, hexane, or dichlorobenzene. In the method, only three chemicals, silver nitrate, oleylamine, and liquid paraffin, were employed throughout the whole process. However, these reactions possess many problems: a large volume of organic solvent or expensive reagents are needed; the reduction process proceeds at relatively high temperature; and the complete separation of silver particles is not easy because of the presence of other compounds in the reaction mixture. Green approaches are the choice of the solvent medium (preferably water), an environmentally friendly reducing agent, and a nontoxic material for the stabilization of the nanoparticles. To be energy efficient, the synthesis processes should be carried out close to ambient temperature and pressure and under neutral $\mathrm{pH}$.

In order to achieve this aim, we have developed simple methods to synthesize AgNPs with high electrical conductivity in the past few years. The synthesis approaches provided ease of purification by using low molecular and without any organic solvent, and operation at room temperature. In this review, we give some description for the preparation, characteristics, and properties of silver nanoparticles according to our recent studies. In addition, we also discuss the reaction mechanism and the factors affecting particle size.

\subsubsection{Synthesis of AgNPs-A Using Aqueous Polymer (Method 1)}

Pure, uniform, and stable colloidal silver nanoparticles can be obtained easily using PVP as a dispersant. AgNPs-A was prepared by the following steps: (1) PVP was dissolved in deionized water by stirring at room temperature. (2) $\mathrm{AgNO}_{3}$ was added and then the solution was stirred further. (3) An aqueous solution of $\mathrm{Na}_{3} \mathrm{Ct}$ in deionized water was added dropwise. (4) After all of the $\mathrm{Na}_{3} \mathrm{Ct}$ solution had been added, an aqueous solution of DMAE in deionized water was added to the reaction mixture, which was then stirred at room temperature for one hour. (5) The silver particles were separated from the solution by centrifugation, washed twice with deionized water, and then redispersed in deionized water. This method has significant advantages: it has a short reaction time; small and relatively uniform particles with a diameter less than $10 \mathrm{~nm}$ are produced; the reaction proceeds rapidly at room temperature; organic solvents are not used; and the resulting particles are easily separated from the reaction mixture.

This method allows uniform AgNPs-A with high purity to be prepared easily. Moreover, by using PVP as a dispersant, pure, uniform, and stable colloidal silver nanoparticles covered with polymer PVP can be obtained easily in very small size, because the dispersant prevents aggregation of the silver nanoparticles. Therefore it is useful for industrial manufacture. However, PVP used for protecting AgNPs affects their electrical conductivity because the resin covered on nanoparticles has to be removed to obtain the electrical conductivity. The sintering process is desired to be performed at low temperature. The volume resistivity of AgNPs-A decreases with rising temperature. The AgNPs-A indicates poor electrical conductivity when they are sintered at less than $200^{\circ} \mathrm{C}$. However, the value of volume resistivity falls largely when the sample is sintered at $250^{\circ} \mathrm{C}$. This can be explained that AgNPs-A contact or fuse each other because the polymer covered on nanoparticles is removed, resulting in good conductivity. The samples using AgNPs-A prepared in our work show a volume resistivity of $1.7 \times 10^{-5}$ at $250^{\circ} \mathrm{C}$, and $6.9 \times 10^{-6} \Omega \cdot \mathrm{cm}$ at $300^{\circ} \mathrm{C}$ which is near to that of metallic silver $\left(1.6 \times 10^{-6} \Omega \cdot \mathrm{cm}\right)$. As a result, AgNPs-A exhibit electroconductivity that is almost as high as that of bulk silver [47]. 


\subsubsection{Synthesis of AgNPs-B Using Low Molecular Weight}

Compounds as Effective Dispersing Agents (Method 2)

By the similar procedure to method 1, AgNPs-B was prepared easily by method 2 . In this method, sodium gluconate or DL-Malic acid disodium salt was used as dispersant instead of PVP. Because sodium gluconate is low molecular weight compounds and water-soluble, the formed AgNPs-B can be separated easily from the mixture. Furthermore, there is no great influence on the particle size with the amount of dispersing agents, which is very different from the case of method 1. Table 1 lists the results of different properties between methods 1 and 2 .

On the other hand, instead of PVP, sodium gluconate can be used as a dispersant to decrease sintering temperature. The comparison of AgNPs volume resistivity between methods 1 and 2 is also shown in Table 1. Compared with AgNPs-A prepared by method 1, AgNPs-B prepared by method 2 are smaller and more uniform in particle size, and exhibit almost the same level of electrical conductivity when sintered at $150^{\circ} \mathrm{C}$ as those of AgNPs-A prepared by method 1 when sintered at $200^{\circ} \mathrm{C}$ [48].

\subsubsection{One-Step Synthesis of AgNPs-C Sintered at Temperature (Method 3)}

Furthermore, we developed a novel method to synthesize AgNPs-C by one-step. In method 3, benzoic acid with over two hydroxyl group such as 3, 4-dihydroxybenzoic acid, gallic acid and dodecyl gallate were used as reducing agents, and AgNPs-C are prepared by varying the amounts of reactants using the similar reaction procedure described above. In this approach to synthesize AgNPs-C, the significant advantages are that any organic solvent and external dispersing agent are not used. Moreover, it is found that the resulting nanoparticles by this procedure can be sintered at room temperature only by drying the solvent within a short time, giving high electrical conductivity [49].

Average particle size and volume resistivity of AgNPs-C prepared by different amines and reducing agent are shown in Table 2. The result indicates that the obtained AgNPs-C shows small size and high electrical conductivity in every case, and amine or reducing agent have some influences on the size of AgNPs-C. The significant advantage of this method is that the resulting nanoparticles by this procedure can be sintered at room temperature only by drying the solvent within a short time, giving high electrical conductivity. This is very important in industry and medical applications.

\section{Characterization of AgNPs and Their Properties}

Characterization of AgNPs is important to understand and control nanoparticles synthesis and applications. Various techniques are used for determination of different parameters.

The morphology of AgNPs is obtained using transmission and scanning electron microscopy (TEM, SEM). The size distribution of AgNPs can be measured with a Zetasizer
Nano Series analyzer. Energy dispersive X-ray spectroscopy (EDS) measurements are used with an emission scanning electron microscope equipped with an EDS instrument. X-ray photoelectron spectroscopy (XPS), X-ray diffractometry (XRD), Fourier transform infrared spectroscopy (FTIR), and UV-Vis spectroscopy are also used to characterize AgNPs. UV-Vis spectroscopy is used to confirm AgNPs formation by showing the Plasmon resonance. Moreover, XRD is used for the determination of crystallinity. The volume resistivity is measured with Loresta-GP MCP-T610 resistivity meter to evaluate electrical conductivity.

For example, in our recent studies, from the results of UVVis measurement for the synthesized AgNPs, optical absorption peak of AgNPs due to surface plasmon resonance is observed [50]. From EDS analysis, the intense peak at around $3 \mathrm{keV}$ is characteristic of AgNPs. No impurities are observed besides small amounts of carbon and oxygen, which indicates that the reagents used have not remained. Because the chemicals used in this study are water-soluble, the final product, which is insoluble in water, can be separated easily from the reaction mixture [47]. XPS evaluation is carried out to determine the elemental composition of AgNPs. The binding energies of $\mathrm{Ag}(3 \mathrm{~d} 3 / 2)$ and $\mathrm{Ag}(3 \mathrm{~d} 5 / 2)$ for AgNPs are observed at $374 \mathrm{eV}$ and 368 $\mathrm{eV}$, respectively. Since the reported binding energy of $\mathrm{Ag} 0$ $(3 \mathrm{~d} 5 / 2)$ is $368.3 \mathrm{eV}$ [51], these peaks prove the formation of AgNPs. Average particle size and volume resistivity of AgNPs are shown in Tables 1 and 2.

\section{Factors Affecting Particle Sizes}

The factors affecting the sizes of AgNPs were also investigated. Various studies were performed where the dispersants, reducing agent, and amine as well as their amounts were varied. Furthermore, the influence of temperature on the size was also investigated.

\subsection{The Size of AgNPs}

The size of AgNPs was obtained by TEM, and the diameter distribution of AgNPs was measured with Zetasizer Nano Series (Malvern Instruments). The distribution ranges of AgNPs-A are approximately from $7 \mathrm{~nm}$ to $40 \mathrm{~nm}$ (Table 1). Although a few larger particles (about $0.8 \%$ ) exist, the diameter of the majority of particles (99.2\%) is less than 10 $\mathrm{nm}$. This indicates that the synthesized AgNPs-A are a narrow distribution of diameters and the average diameter of approximately $10 \mathrm{~nm}$ [47]. AgNPs-B (method 2) have smaller size and narrower distribution than AgNPs-A. On the other hand, the size of AgNPs-C prepared by method 3 is shown in Table 2, small size and relatively uniform particles with a diameter of $10 \mathrm{~nm}$ or less can be obtained under different experimental conditions.

\subsection{The Influence of Dispersants on AgNPs}

Polyvinylpyrrolidone (PVP) plays an important role as a surfactant in the formation of AgNPs. PVP used as dispersant 
can protect nanoparticles in AgNPs preparation because it covers the surface of nanoparticles to form stable colloid, which is useful for industrial manufacture. The amount of PVP as dispersant has an influence on the size of AgNPs. When only a small amount of PVP is used, agglomeration takes place as a result of incomplete covering of AgNPs with PVP. An appropriately weight ratio of $\mathrm{PVP} / \mathrm{AgNO}_{3}$ should be an optimum to produce small sized AgNPs. In this case, the entire surface of AgNPs is coated with PVP. Consequently, the agglomeration is prevented by PVP keeping the silver particles separated. However, the size of the metal nanoparticles increases when excess PVP is added. This indicates that excess PVP does not further reduce the size of the colloid. Excess PVP does not appear to provide better coverage of the surface of the silver colloid, or it may hinder the formation of the nanoparticles. Fourier transform infrared absorption spectroscopy (FTIR) spectra show that the silver particles coordinate with $\mathrm{N}$ or $\mathrm{O}$ in PVP, generating a cover on the surface of the metal particles. The layer can prevent the silver particles from agglomerating, and consequently produce spherical colloidal particles [47].

Possible protection and the reaction mechanism of sodium gluconate $\left(\mathrm{C}_{5} \mathrm{H}_{11} \mathrm{O}_{5} \mathrm{COONa}\right)$ as dispersing agent are also demonstrated using FTIR analysis. In this case, $\mathrm{C}_{5} \mathrm{H}_{11} \mathrm{O}_{5} \mathrm{COO}^{-}$, generating a cover on the surface of the metal particles is confirmed. The layer can prevent AgNPs from agglomerating, leading to small spherical colloidal particles. It seems that the charges on the surface of nanoparticles do not increase with the amount of dispersing agents. This is different from the neutral polymeric compounds, in which particle size is affected easily by the amount of dispersing agents since the nanoparticles are dispersed by steric hindrance of PVP [48].

Therefore Compared with AgNPs-A formed using PVP as the dispersing agent, there is no great influence on the particle size in AgNPs-B whether sodium gluconate or DLMalic acid disodium salt is used as the dispersing agent (Table 1).

Table 1. Comparison of the properties of $A g N P s-A$ and $A g N P s-B$.

\begin{tabular}{lllll}
\hline $\begin{array}{l}\text { Silver } \\
\text { nanoparticles }\end{array}$ & $\begin{array}{l}\text { Dispersing } \\
\text { agent }\end{array}$ & $\begin{array}{l}\text { Average } \\
\text { particle } \\
\text { size }(\mathbf{n m})\end{array}$ & $\begin{array}{l}\text { Sintering } \\
\text { temperature } \\
\left({ }^{\circ} \mathbf{C}\right)\end{array}$ & $\begin{array}{l}\text { Volume } \\
\text { resistivity } \\
(\boldsymbol{\Omega} \cdot \mathbf{c m})\end{array}$ \\
AgNPs-A & PVP & $5-40$ & 200 & $1.6 \times 10^{-4}$ \\
AgNPs-B & $\begin{array}{l}\text { Sodium } \\
\text { gluconate }\end{array}$ & $4-10$ & 150 & $4.6 \times 10^{-4}$ \\
\hline
\end{tabular}

\subsection{The Influence of Amines}

The amine plays an important role in the reduction of silver ions in aqueous solution. $\mathrm{Na}_{3} \mathrm{Ct}$ can be used not only as a dispersing agent, but also as a reducing agent. However, it is a weak reductant, and its reducing ability is dependent on the $\mathrm{pH}$ of the solution. When $\mathrm{Na}_{3} \mathrm{Ct}$ alone is used as a reducing agent, higher temperatures are usually required to promote reduction $\left(100^{\circ} \mathrm{C}\right)$. In our study, however, the reactions occur rapidly at room temperature by addition of DMAE. The reaction mechanism of AgNPs fabricated using dispersing agent (DA) can be supposed as follows:

$$
\begin{gathered}
\text { DMAE }+\mathrm{H}_{2} \mathrm{O} \rightarrow \mathrm{DMAE}^{+}+\mathrm{OH}^{-} \\
\mathrm{Ag}^{+}+\mathrm{DA} \rightarrow \mathrm{Ag}(\mathrm{DA})^{+} \\
\mathrm{Na}_{3} \mathrm{Ct}(\mathrm{C}-\mathrm{OH})+2 \mathrm{OH}^{-} \rightarrow \mathrm{Na}_{3} \mathrm{Ct}(\mathrm{C}=\mathrm{O})+\mathrm{H}_{2} \mathrm{O}+2 \mathrm{e}^{-} \\
\mathrm{Ag}(\mathrm{DA})^{+}+\mathrm{e}^{-} \rightarrow \mathrm{Ag}(\mathrm{DA})+\mathrm{DMAE}
\end{gathered}
$$

As shown in Eq. (1), when the amine is dissolved in water, it withdraws a hydrogen ion and leaves a hydroxyl ion in solution. The hydroxyl ions oxidize the $\mathrm{OH}$ group in $\mathrm{Na}_{3} \mathrm{Ct}$ and an electron is released in the process. It is thought that the amine acts as a catalyst that can accelerate the reaction.

Moreover, in this case of AgNPs-C using benzoic acid as dispersing agent, it is clear that benzoic acid plays a role in not only reducing agent but also stabilizing agent in this process. Other amines were also examined. It is found that smaller AgNPs-C can be obtained under relatively weak alkaline conditions (Table 2 and see [49]).

Table 2. Average particle size and volume resistivity of $\mathrm{AgNPs}-\mathrm{C}$ prepared by different amines and reducing agent.

\begin{tabular}{lllll}
\hline $\begin{array}{l}\text { AgNP } \\
\text { s-C }\end{array}$ & Amine & $\begin{array}{l}\text { Reducing } \\
\text { agent }\end{array}$ & $\begin{array}{l}\text { Average particle } \\
\text { size }(\mathbf{n m})\end{array}$ & $\begin{array}{l}\text { Volume resistivity } \\
(\mathbf{\Omega} \cdot \mathbf{c m})\end{array}$ \\
\hline 1 & $\mathrm{Et}_{3} \mathrm{~N}$ & Gallic acid & 10 & $5.6 \times 10^{-4}$ \\
2 & $\mathrm{DMAE}$ & $\begin{array}{l}\text { Gallic acid } \\
\text { Dodecyl } \\
\text { gallate }\end{array}$ & 3 & $5.1 \times 10^{-4}$ \\
3 & $\mathrm{Et}_{3} \mathrm{~N}$ & $\begin{array}{l}\text { Dihydroxyben } \\
\text { zoic acid }\end{array}$ & 8 & $1.7 \times 10^{-2}$ \\
4 & $\mathrm{Et}_{3} \mathrm{~N}$ & $\begin{array}{l}\text { zoic } \\
4\end{array}$ \\
\hline
\end{tabular}

\subsection{The Effect of Temperature}

As described in our previous work, when the reaction was performed at $50^{\circ} \mathrm{C}$, the reaction mixture quickly turned brown, and a black powder was collected as the final product. TEM image of AgNPs showed that large silver particles have formed and the particles are not spherical, appearing to be linked. This probably results from coordination among silver particles surface covered with PVP at high temperature. This indicates that the reaction rate increased significantly, resulting in the formation of larger particles and the occurrence of an undesirable reaction when the reaction is performed at high temperature. On the other hand, when the reaction was performed at $10^{\circ} \mathrm{C}$, the rate of reaction was very slow and the color of the solution began to change only after $3 \mathrm{~h}$ [47].

\section{Applications of AgNPs}

Silver nanoparticles are one of the most attractive nanomaterials for commercialization applications. They have been used extensively as electronic products in the industry, anti-bacterial agents in the health industry, food storage, textile coatings and a number of environmental applications. As anti-bacterial agents, silver nanoparticles were used for a wide range of applications from disinfecting medical devices and home appliances to water treatment [52-55]. Moreover, this encouraged the textile industry to use AgNPs in different 
textile fabrics. In this direction, silver nanocomposite fibers were prepared containing AgNPs incorporated inside the fabric [56]. The cotton fibers containing AgNPs exhibited high anti- bacterial activity against Escherichia coli [56, 57]. Silver nanoparticles were found to catalyze the chemiluminescence from luminol-hydrogen peroxide system with catalytic activity better than Au and Pt colloid [58].

Recently, inkjet technology has been used to produce flexible electronic circuits at low cost, and many studies regarding this application have been reported in recent years [23-25]. To fabricate flexible electronic displays via inkjet printing, it is necessary to develop suitable inks. Nano-sized metal particles such as $\mathrm{Au}$ or $\mathrm{Ag}$ are useful for producing electronic circuits because of the uniformity of the small metal particles dispersed in the inks and their high electrical conductivity. For example, using our methods described above, AgNPs with small size and uniform can be prepared easily, and have high electrical conductivity, indicating that they are useful for producing electronic circuits. In the manufacture of electronic circuits, nanoparticles must be sintered to obtain high electrical conductivity. It is preferable to perform sintering at the lowest temperature possible. However, the use of polymeric materials as dispersing agents means that a high temperature is required for sintering.

In our method 1, polymeric compound PVP was used as a dispersing agent in the synthesis of AgNPs-A. The use of PVP with high molecular weight resulted in a high temperature $\left(>200^{\circ} \mathrm{C}\right)$ being required for sintering. In contrast, low molecular weight compounds such as sodium gluconate have good protecting function similar to polymeric compounds, which can produce uniform, small AgNPs-B with high conductivity and also allow them to be sintered at a relatively low temperature of $150^{\circ} \mathrm{C}$ (method 2). The lowering of the sintering temperature in 50 degrees is very important and useful from the perspective of industrial manufacturing. In particular, a simple approach to synthesize AgNPs is developed using benzoic acid and amines as reducing agents (method 3). A film with high electrical conductivity can be obtained using AgNPs-C prepared only by drying at room temperature. This is very important to prepare silver nanoparticles in industry and medical applications.

\section{Conclusion}

In this review, different methods to prepare AgNPs and their properties as well as applications are presented. In particular, several novel chemical methods based on our recent studies are described, which are successful in the synthesis of AgNPs with high conductive properties. The reaction mechanism of AgNPs and factors affecting particle size are also clarified. Significant advantages of these methods over previous ones include: it has a short reaction time; relatively uniform particles with small diameter are produced; the reaction proceeds rapidly at room temperature; organic solvents are not used, and used chemical reagents are water soluble, cheap, easy to deal with, not producing hazardous by-products and environmentally friendly; and the resulting particles are easily separated from the reaction mixture. Therefore, these approaches can contribute to saving energy and reduce the cost of preparing AgNPs. These methods are also safe and environmentally benign, which are very important factors from the perspective of industrial manufacturing. Particularly, these advantages of these methods are very important to use AgNPs for medical applications because of non-toxicity.

Therefore, these advantages make the present methods practically useful and potentially applicable to large-scale industrial manufacture of stable colloids silver nanoparticles, which are applicable in various fields, especially digital fabrication of electronic circuits and medicial applications.

\section{Acknowledgment}

This work was supported by JSPS KAKENHI Grant Number 10432171.

\section{References}

[1] G. Franci, A. Falanga, S. Galdiero, L. Palomba, M. Rai, G. Morelli, M. Galdiero, "Silver nanoparticles as potential antibacterial agents", Molecules, vol.20, pp.8856-8874, 2015.

[2] S. Jana, T. Pal, "Synthesis, characterization and catalytic application of silver nanoshell coated functionalized polystyrene beads", J. Nanosci. Nanotechnol. vol.7, pp.21512156, 2007

[3] R. Stiufiuc, C. Iacovita, C. M. Lucaciu, G. Stiufiuc, A. G. Dutu, C. Braescu, N. Leopold, "SER-sactive silver colloids prepared by reduction of silver nitrate with short-chain polyethylene glycol”, Nanoscale Res. Lett. vol.8, pp.47, 2013.

[4] M. P. Konrad, A. P. Doherty, S. E. J. Bell, "Stable and uniform SERS signals from self-assembled two-dimensional interfacial arrays of optically coupled Ag nanoparticles", Anal. Chem. vol.85, pp.6783-6789, 2013.

[5] G. M. Meheretu, D. Cialla, J. Popp, "Surface enhanced raman spectroscopy on silver nanoparticles", Inter. J. Biochemistry Biophysics, vol.2, pp.63-67, 2014.

[6] G. A. Evtugyn, R. V. Shamagsumova, P. V. Padnya, I. I. Stoikov, I. S. Antipin, "Cholinesterase sensor based on glassy carbon electrode modified with Ag nanoparticles decorated with macrocyclic ligands", Talanta, vol.127, pp.9-17, 2014.

[7] N. T. K. Thanha, L. A. W. Green, "Functionalisation of nanoparticles for biomedical applications", Nano Today, vol.5, pp.213-230, 2010.

[8] N. Alon, Y. Miroshnikov, N. Perkas, I. Nissan, A. Gedanken, O. Shefi, "Substrates coated with silver nanoparticles as a neuronal regenerative material", Int. J. Nanomed. vol.9, pp.23-31, 2014.

[9] Y. Bu, S. Lee, "Influence of dopamine concentration and surface coverage of $\mathrm{Au}$ shell on the optical properties of $\mathrm{Au}$, $\mathrm{Ag}$, and AgcoreAushell nanoparticles", ACS Appl. Mater. Interfaces vol.4, pp. 3923-3931, 2012. 
[10] Y. Luo, L. Ma, X. Zhang, A. Liang, Z. Jiang, "SERS detection of dopamine using label-free acridine red as molecular probe in reduced graphene oxide/silver nanotriangle sol substrate", Nanoscale Research Letters, vol.10, pp.230, 2015.

[11] P. J. Rivero, A. Urrutia, J. Goicoechea, I. R. Matias, F. J. Arregui, "A Lossy Mode Resonance optical sensor using silver nanoparticles-loaded films for monitoring human breathing", Sens. Actuators B, vol.187, pp.40-44, 2013.

[12] S. Tsuruga, T. Abe, "Preparation of electro-conductive inkjet inks through silver halide photographic emulsion", Proceedings of the Pan-Pacific Imaging Conference, pp.56-59, 2008.

[13] S. Iwama, T. Sahashi, "Sintering of ultrafine metal powders. I. Coalescence growth stage of Au and Ag", Japn. J. Appl. Phys. vol.19, pp.1039-1044, 1980.

[14] I. Sondi, D. V. Goia, E. Matijevic, "Preparation of highly concentrated stable dispersions of monodispersed silver nanoparticles", J. Colloid. Interface Sci. vol.260, pp. 75-81, 2003.

[15] K. M. M. El-Nour, A. Eftaiha, A. Al-Reda, A. A. Ammar, "Synthesis and applications of silver nanoparticles", Arabian J. Chem. vol.3, pp.135-140, 2010.

[16] A. B. Smetana, K. J. Klabunde, C. M. Sorensen, "Synthesis of spherical silver nanoparticles by digestive ripening, stabilization with various agents, and their 3-D and 2-D superlattice formation", J. Colloid. Interface Sci. vol.284, pp. 521-526, 2005.

[17] D. Wakuda, K.S. Kim, K. Suganuma, "Room temperature sintering of Ag nanoparticles by drying solvent", Scrip. Mater. vol.59, pp.649-652, 2008.

[18] H. Lee, K. S. Chou, "Inkjet Printing of Nanosized Silver Colloids", Nanotechnology, vol.16, pp.2436-2441, 2005.

[19] Z. Anna, S. Ewa, Z. Adriana, G. Maria, H. Jan, "Preparation of silver nanoparticles with controlled particle size", Procedia Chem. vol.1, pp.1560-1566, 2009.

[20] S. Iravani, H. Korbekandi, S. V. Mirmohammadi, B. Zolfaghari, "Synthesis of silver nanoparticles: chemical, physical and biological methods", Research in Pharmaceutical Sciences, vol.9, pp.385-406, 2014.

[21] M. M. Kholoud, A. E. Nour, A. Eftaiha, A. A. Warthan, and R. A. A. Ammar, "Synthesis and applications of silver nanoparticles", Arabian Journal of Chemistry, vol.3, pp.135140, 2010.

[22] K. Toisawa, Y. Hayashi, H. Takizawa, "Synthesis of highly concentrated Ag nanoparticles in a heterogeneous solid-liquid system under ultrasonic irradiation”, Materials Transactions, vol.51, pp.1764-1768, 2010.

[23] A. lennon, R. Utama, A. Ho-Baillie, M. Lenio, N. Kuepper, S. Wenham, in: Proceedings of the NIP 23 and Digital Fabrication Conference, 2007.

[24] A. lennon, R. Utama, A. Ho-Baillie, and S. Wenham, Proceedings of the NIP 24 and Digital Fabrication Conference, 2008.

[25] S. Tsuruga, and T. Abe, "Preparation of electro-conductive inkjet inks through silver halide photographic emulsion", Proceedings of the Pan-Pacific Imaging Conference, pp.56-59, 2008.
[26] H. H. Lee, K. S. Chou, K. C. Huang, "Inkjet printing of nanosized silver colloids", Nanotechnology vol.16, pp.24362441, 2005.

[27] B. C. Gates, "Supported Metal Clusters: Synthesis, Structure, and Catalysis", Chem. Rev. Vol.95, pp.511-522, 1995.

[28] N. R. Jana, T. K. Sau, T. J. Pal, "Growing Small Silver Particles as Redox Catalyst", J. Phys. Chem. B vol.103, pp.115-121, 1999.

[29] A. S. Gurav, T. T. Kodas, L. M. Wang, E. I. Kauppinen, and J. Joutsensaari "Generation of nanometer-size fullerene particles via vapor condensation", J. Joutsensaari Chem. Phys. Lett. vol.218, pp.304-308, 1994.

[30] F. Kruis, H. Fissan, B. Rellinghaus, "Sintering and evaporation characteristics of gas-phase synthesis of sizeselected PbS nanoparticles", Mater. Sci. Eng. B vol.69-70, pp.329-334, 2000.

[31] M. H. Magnusson, K. Deppert, J. O. Malm, J. O. Bovin, L. Samuelson, "Gold nanoparticles: Production, reshaping, and thermal charging", J. Nanoparticle Res. vol.1, pp.243-251, 1999.

[32] F. Mafune, J. Kohno, Y. Takeda, T. Kondow, H. Sawabe, "Formation and size control of silver nanoparticles by laser ablation in aqueous solution", J. Phys. Chem. B, vol.104, pp.9111-9117, 2000.

[33] I. Dolgaev, A. V. Simakin, V. V. Voronov, G. A. Shafeev, F. B. Verduraz, Nanoparticles producedby laser ablation of solids in liquid environments, Appl. Surf. Sci. vol.186, pp.546-551, 2002.

[34] J. P. Sylvestre, A. V. Kabashin, E. Sacher, M. Meunier, J. H. T. Luong, "Stabilization and size control of gold nanoparticles during laser ablation in aqueous cyclodextrins", J. Am. Chem. Soc. vol.126, pp.7176-7177, 2004.

[35] T. Tsuji, K. Iryo, N. Watanabe, M. Tsuji, "Preparation of silver nanoparticles by laser ablation in solution: Influence of laser wavelength on particle size" Appl. Surf. Sci. vol.202, pp.8085. 2002.

[36] H. Huang, Y. Yang, "Preparation of silver nanoparticles in inorganic clay suspensions", Compos. Sci. Technol. vol.68, pp.2948-2953, 2008.

[37] A. R. Vilchis-Nestor, V. nchez-Mendieta, M. A. CamachoLo'pez, R. M. Go'mez-Espinosa, M. A. Camacho-Lo'pez, J. A. Arenas-Alatorre, "Solventless synthesis and optical properties of $\mathrm{Au}$ and $\mathrm{Ag}$ nanoparticles using Camellia sinensis extract", Mater. Lett. vol.62, pp.3103-3105, 2008.

[38] K. Kalishwaralal, V. Deepak, S. Ramkumarpandian, H. Nellaiah, and G. Sangiliyandi, "Extracellular biosynthesis of silver nanoparticles by the culture supernatant of Bacillus licheniformis", Mater. Lett. vol. 62, pp.4411-4413, 2008.

[39] S. F. Chen, H. Zhang, Adv. Nat. Sci.: Nanosci. Nanotechnol. "Aggregation kinetics of nanosilver in different water condition", vol.3, pp.035006-1-7, 2012.

[40] T. M. D. Dang, T. T. T. Le, E. F. Blance, M. C. Dang, "Influence of surfactant on the preparation of silver nanoparticles by polyol method", Adv. Nat. Sci.: Nanosci. Nanotechnol. vol.3, pp.035004-1-4, 2012. 
[41] R. S. Patil, M. R. Kokate, C. Jambhale, S. M. Pawar, S. H. Han, S. S. Kolekar, "One-pot synthesis of PVA-capped silver nanoparticles their characterization and biomedical application", Adv. Nat. Sci.: Nanosci. Nanotechnol. vol.3, pp.015013-1-7, 2012.

[42] Q. H. Tran, V. Q. Nguyen, A. T. Le, "Silver nanoparticles: synthesis, properties, toxicology, applications and perspectives", Adv. Nat. Sci. Nanosci. Nanotechnol. vol.4, pp.033001-1-19, 2013.

[43] D. Radziuk, A. Skirtach, G. Sukhrukov, D. Shchukin, "Stabilization of silver nanoparticles by polyelectrolytes and poly (ethylene glycol)", Macromol: Rapid. Commun. vol.28, pp.848-855, 2007.

[44] C. Luo, Y. Zhang, X. Zeng, Y. Zeng, Y. Wang, "The role of poly(ethylene glycol) in the formation of silver nanoparticles", J. Colloi. Interface Sci. vol. 288(2), pp.444-448, 2005.

[45] S. L. C. Hsu, R. T. Wu, "Synthesis of contamination-free silver nanoparticle suspensions for micro-interconnects", Mater. Lett. vol.61, pp.3719-3722, 2007.

[46] M. Chen, Y. G. Feng, X. Wang, T. C. Li, J. Y. Zhang, D. J. Qian, "Silver nanoparticles capped by oleylamine: formation, growth, and self-organization", Langmuir, vol.23, pp.52965304, 2007.

[47] J. Natsuki, T. Abe, "Synthesis of pure colloidal silver nanoparticles with high electroconductivity for printed electronic circuits: The effect of amines on their formation in aqueous media", J. Colloid. Interf. Sci. vol.359, pp.19-23, 2011.

[48] J. Natsuki, T. Natsuki, T. Abe, "Low molecular weight compounds as effective dispersing agents in the formation of colloidal silver nanoparticles", J. Nanopart. Res. vol.15, pp.1483-1-8, 2013.

[49] J. Natsuki, T. Natsuki, "One-step synthesis of silver nanoparticles using low molecular weight compounds at room temperature", Inter. J. Mater. Eng. Techno. vol.13, pp.109-119, 2015.
[50] K. S. Chou, Y. S. Lai, "Effect of polyvinyl pyrrlidone molecular weights on the formation of nanosized siver colloids" Mater. Chem. Phys. vol.83, pp.82-88, 2004.

[51] S. He, J. Yao, S. Xie, H. Gao, S. Pang, "Superlattices of silver nanoparticles passivated by mercaptan", J. Phys. D. vol.34, pp.3425-3429, 2001.

[52] M. Bosetti, A. Masse, E. Tobin, M. Cannas, "Silver coated materials for external fixation devices: in vitro biocompatibility and genotoxicity", Biomaterials vol.23, pp.887-892, 2002.

[53] P. Jain, T. Pradeep, "Potential of silver nanoparticle-coated polyurethane foam as an antibacterial water filter", Biotechnol. Bioeng. vol.90, pp.59-63, 2005.

[54] Q. Li, S. Mahendra, D. Y. Lyon, L. Brunet, M. V. Liga, D. Li, P. J. Alvarez, "Antimicrobial nanomaterials for water disinfection and microbial control: potential applications and implications", Water Res. vol.42, pp.4591-4602, 2008.

[55] M. Cho, H. Chung, W. Choi, J. Yoon, "Different inactivation behaviors of MS-2 phage and Escherichia coli in $\mathrm{TiO} 2$ photocatalytic disinfection", Appl. Environ. Microbiol. vol.71, pp.270-275, 2005.

[56] S. Y. Yeo, H. J. Lee, S. H. Jeong, "Preparation of nanocomposite fibers for permanent antibacterial effect", J. Mater. Sci. vol.38, pp.2143-2147, 2013.

[57] N. Duran, P. D. Marcarto, G. H. D. Souza, O. L. Alves, E. Esposito, "Antibacterial effect of silver nanoparticles produced by dungal process on textile fabrics and their effluent treatment”, J Biomed Nanotechnol vol.3, pp.203-208, 2007.

[58] J. Z. Guo, H. Cui, W. Zhou, W. Wang, "Ag nanoparticlecatalyzed chemiluminescent reaction between luminol and hydrogen peroxide", J. Photochem. Photobiol. A: Chem. vol.193, pp.89-96, 2008. 